\title{
Semantic processing of unattended text during selective reading: How the eyes see it
}

\author{
ALBRECHT WERNER INHOFF and DEBORAH BRIIHL \\ State University of New York at Binghamton, Binghamton, New York
}

\begin{abstract}
Subjects were instructed to read and comprehend a target (attended) passage while eye movements were recorded. A second (unattended) passage was also present, with attended and unattended passages occupying alternating lines of text. Subsequent multiple-choice questions showed acquisition of semantic information from attended and unattended text. However, a detailed examination of eye-fixation records showed that readers occasionally fixated unattended text, in dicating the presence of shifts of visual attention to unattended text. When fixations of unattended text were excluded, there was no longer any indication that readers obtained useful semantic information from unattended text.
\end{abstract}

The representation of unattended text under selective attention conditions has been of considerable interest. Most studies converge in showing that physical characteristics of the stimulus source can be ascertained prior to the orienting of attention; there is considerable controversy, however, about whether or not unattended text can be represented semantically. Several studies in the auditory domain indicate that this may be the case (e.g., Corteen \& Dunn, 1974; Corteen \& Wood, 1972; Lackner \& Garrett, 1972; Lewis, 1970; MacKay, 1973; Treisman, 1960; Treisman, Squire, \& Green, 1974; Von Wright, Anderson, \& Stenman, 1975; Zelnicker, 1971). In a frequently quoted study, Treisman (1960) showed that listeners' shadowing switched occasionally from the attended to the unattended channel when text in the unattended channel, rather than the attended channel, provided a meaningful continuation of text. This occurred, presumably, because subjects had some knowledge of the semantic content of nonshadowed text.

Analogous results have been obtained in a selective reading task, in which subjects were instructed to read lines of attended text that alternated with lines of tobe-neglected (unattended) text (Willows \& MacKinnon, 1973). Even though readers in the experiments were not aware of the meaning expressed in unattended lines, they seemed to use semantic information from these lines to answer subsequent questions pertaining to unattended text. Results from auditory and visual domains thus appear to

\footnotetext{
This research was supported by Grant HD24796 from the National Institute of Child Health and Human Development and by Grant BNS9010067 from the National Science Foundation. I would like to thank Cynthia Connine, Philip Merikle, Tram Neill, Charles Eriksen, and an anonymous reviewer for their helpful comments on an earlier version of the manuscript. Special thanks to Charles Clifton at the University of Massachusetts for the use of his software. Requests for reprints, as well as other correspondence, should be sent to Albrecht Inhoff, Department of Psychology, SUNY-Center Binghamton, State University of New York, Binghamton, NY 13902-6000.
}

converge to show that unattended text is represented in a semantic format.

However, many of these studies suffer from methodological shortcomings. They rely on the assumptions that the monitoring of the to-be-attended source is continuous and uninterrupted and that the monitoring remains at a constant level of "commitment." Yet, given that dichotic listening and selective reading generally extend over a relatively long time span, these assumptions may not be warranted. Consequently, it cannot be ruled out that any evidence in support of the semantic representation of unattended text is artifactual, resulting from temporary switches of attention to the unattended (or to-be-neglected) source (see Holender, 1986, for an elaboration of this view). Consider, for instance, Treisman's (1960) classic result: As Cowan (1988) pointed out, subjects may have shifted attention to the unattended channel, because the attended channel suddenly changed from a coherent to an incoherent passage. Analogously, subjects may have shifted visual attention to unattended lines in Willows and MacKinnon's selective reading (1973) experiments.

Quite consistent with this view, some well controlled studies in the auditory domain showed that unattended (unshadowed) information is not represented in a semantic format when attention is focused on the attended channel (e.g., Johnston \& Wilson, 1980; Newstead \& Dennis, 1979). Furthermore, one study that used auditorily presented stimuli failed to replicate crucial aspects of earlier findings that had seemingly demonstrated the semantic representation of unattended words (Wardlaw \& Kroll, 1976). It also remains unclear whether or not Willows and MacKinnon's (1973) findings indicate that readers obtained semantic information from unattended text in selective reading. Readers could have shifted attention to unattended text, which could have resulted in the acquisition of effective semantic information. Even if these shifts of attention occurred rarely and were difficult to recall, they could have biased readers' performance on the subsequent recognition test. 
It is difficult, if not impossible, to find a direct measure of indexing shifts of attention from attended to unattended text in the auditory domain. In selective reading, however, shifts of visual attention from a to-be-attended source to a to-be-neglected source may be accompanied by corresponding shifts of eye position (Morrison, 1984). If this were the case, then measurements of eye positions during selective reading could be used to determine, first, whether or not spontaneous shifts of attention to unattended text occur during selective reading and, second, whether or not unattended text is semantically represented, given that there was no observable shift of attention. The present study used a selective reading task to examine the prevalence of spontaneous shifts of attention and their effects on the representation of unattended text.

\section{METHOD}

Our study was similar to Willows and MacKinnon's (1973) selective reading experiments, in which subjects were required to read text consisting of alternating lines of attended and to-be-neglected text. Eye fixations were recorded to determine whether or not inadvertent shifts of visuospatial attention to unattended lines took place.

\section{Subjects}

Sixteen undergraduate students at the State University Center of New York at Binghamton participated in the study for course credit. All of the subjects were fluent readers with uncorrected vision.

\section{Apparatus}

Eye movements were recorded with a dual-Purkinje SRI fifth generation eye-tracking system with a visual resolution of approximately $10^{\circ}$ of visual angle. Viewing was binocular, but eye movements were recorded from the right eye only. Text was presented on a SONY multisync monitor that was approximately $35 \mathrm{~cm}$ from the subjects' eyes. At this viewing distance, each character of text subtended, horizontally, approximately $.3^{\circ}$ of visual angle, and the centers of two lines of text were separated vertically by approximately $.6^{\circ}$ of visual angle. All letters appeared in light green on a black background. The monitor, the eye tracking system, and two manual pushbutton boards were interfaced with a Dell 310 microcomputer (equipped with a Data Translation A-to-D converter and a Metrabyte clock) that controlled the experiment. Fixation durations were measured to the nearest millisecond (msec), horizontal fixation locations were measured to the nearest character space, and vertical movements were measured to the nearest line unit.

\section{Materials}

Twelve length-matched passages were constructed, each of which contained between 24 and 36 words. All passages extended across three lines of text, with no line containing more than 72 character spaces. Passages were selected so that they contained specific words that were essential for the answering of subsequently presented multiple-choice questions. Hereafter, we will refer to these critical text locations as targets. Two passages occupied alternating lines of text during each passage reading so that one passage occupied lines 1,3 , and 5 and a second passage occupied lines 2,4 , and 6. Three multiple-choice questions accompanied each passage. All multiple-choice questions contained three or four plausible-choice alternatives. An attended and unattended passage with corresponding multiple-choice questions is shown in the Appendix.

\section{Procedure}

A two-dimensional calibration of the eye tracking system initiated the experiment. After calibration, subjects' fixation location was indicated by a green cursor that moved in synchrony with the subjects' eyes. The accuracy of the tracking system was determined by asking the subject to fixate several prespecified locations on the monitor. A calibration was considered successful when intended and actual fixation positions differed by no more than one character space $\left(.3^{\circ}\right.$ of visual angle). After successful calibration, a fixation marker appeared on the left side of the screen, coinciding with the first letter of the to-be-presented text. When this marker was fixated, the experimenter triggered the display of text (by depressing a pushbutton that was mounted on one of the two response panels).

Two lines of text were presented simultaneously, comprising, for instance, lines 1 and 2 of the two passages, with line 1 comprising the first line of the to-be-attended passage and line 2 comprising the first line of the to-be-neglected passage. Except for to-becapitalized letters, all text on one line comprised lowercase letters; all text on the remaining line comprised uppercase letters. Subjects were instructed to read and to attend to the upper line only. After the upper line was read, the subject depressed a key on the second response panel, which erased the two lines of text and replaced the display with a left-side fixation marker. Upon its successful fixation, the next two lines of attended and unattended text (e.g., lines 3 and 4 ) were presented at the same spatial CRT location as sentences 1 and 2 .

Our text-presentation mode and Willows and MacKinnon's textpresentation mode differed slightly. We used sequentially presented two-line CRT displays, whereas Willows and MacKinnon presented attended and to-be-neglected passages on a single page of text. (We decided to use two-line text displays to minimize track losses, which occur most frequently when readers execute large return sweeps from the end of one line to the beginning of the next line.) In the present study, attended and unattended text was discriminable by means of letter-case and line assignments. Willows and MacKinnon's stimuli were discriminable by means of color and line assignments.

After each attended passage was read, the subject answered six multiple-choice questions, of which three referred to targets of the attended passage and three referred to targets of the unattended passage. On half of the trials, the first three questions probed content from the attended passages and the last three questions probed content from unattended passages; this question ordering was reversed on the remaining trials. The subjects were informed that passage reading should provide correct answers to about half of the questions asked. Readers were encouraged to guess the correct answers for the remaining questions. They were told that this guessing would be used to assess their general level of knowledge.

All of the subjects read two practice passages and answered questions pertaining to these passages prior to the presentation of the experimental passages.

\section{Design}

Four versions of each story pair were constructed. In two versions, one member of the pair was used as an attended passage; in the remaining two versions, this member was used as an unattended passage. Each attended and unattended passage was typed in uppercase in one display mode and in lowercase in another display mode. Whenever the attended passage was typed in lowercase, the unattended passage was typed in uppercase, and vice versa. Four lists of stimulus materials were constructed, each containing a different version of each story pair. List order was counterbalanced across 4 consecutive subjects.

Accuracy in answering multiple-choice questions was the primary dependent variable. Supplementary analyses were applied to fixation durations. 


\section{RESULTS}

\section{Forced-Choice Performance}

Overall, multiple-choice questions were answered almost twice as accurately when the passage was attended $(p=.79)$ than when it was unattended $(p=.41)[t(35)=$ 9.765, $\left.M S_{\mathrm{e}}=37.5, p<.001\right]$. To determine if readers obtained useful semantic information from unattended text, a baseline level of question answering performance needed to be established. Given the number of choice alternatives per question, the a priori probability of answering a multiple-choice question correctly was either $p=$ .25 or $p=.33$, per item. Averaged across items, the probability of guessing correctly was $p=.28$. However, this estimate of chance performance does not take the subjects' prior knowledge into account. To control for this, 22 undergraduate volunteers, who did not participate in the reading study, answered the multiple-choice questions without prior passage reading. Their probability of answering items correctly ranged from $p=0$ to $p=.956$ (for one item). Averaged across items, this empirically determined probability of guessing correctly amounted to $\dot{p}=.31$.

Given this empirically determined baseline level of guessing, passage reading increased the number of correctly answered questions when the passage was attended $\left[t(35)=12.991, M S_{\mathrm{e}}=36.5, p<.001\right]$ and when the passage was unattended $\left[t(35)=3.328, M S_{\mathrm{e}}=3.20\right.$, $p<.002]$. Confirming Willows and MacKinnon's (1973) results, this finding indicates that unattended visual text can be represented semantically. Furthermore, informal questioning of all of the subjects after task completion indicated that none of the subjects had been aware of shifts of attention to the to-be-neglected text. In nearly all instances, the subjects insisted that no effective information had been obtained from the unattended source and that multiple-choice performance was based on "pure" guessing.

However, examination of eye-movement records revealed occasional fixations of unattended text. Twentyseven percent of all unattended lines received at least one fixation; $12.5 \%$ of the unattended targets were the direct recipients of at least one fixation. Even though subjects were unable (or unwilling) to report these shifts of visuospatial attention to the to-be-neglected text, such shifts of attention did occur.

As a consequence of this finding, two supplementary analyses were performed that examined the probability of answering a multiple-choice question correctly, given that the unattended target had been fixated $(58.3 \%$ correct forced-choice performance) and given that the line containing the unattended target had been fixated ( $44.6 \%$ correct performance). No statistical tests were applied, due to the small number of items for which data were available. Nevertheless, these results indicate that the probability of guessing correctly increased modestly when the readers fixated the to-be-neglected line containing the relevant target and increased substantially when the tobe-neglected target was the direct recipient of a fixation.

More importantly, we also analyzed multiple-choice performance when there was no indication of shifts of attention to unattended text. When the relevant unattended target was not directly fixated, the probability of answering a corresponding multiple-choice question correctly amounted to $p=.375$, which was significantly higher than the probability of guessing performance $[t(35)=$ $\left.2.004, M S_{\mathrm{e}}=3.23, p<.05\right]$. However, when the line containing an unattended target was not fixated, the probability of answering the corresponding multiple-choice question correctly amounted to $p=.330$, which no longer differed from guessing performance $(t<1)$. This finding suggests that some semantic information that was obtained during the fixation of the to-be-neglected line was used to constrain guessing in the multiple-choice task. Shifts of visuospatial attention to the unattended line thus preceded (or co-occurred with) the acquisition of useful semantic information. Without such shifts of eye position to unattended text, no effective semantic information was obtained. ${ }^{1}$

It is unlikely that the lack of semantic activation for unattended (and nonfixated) text is due to retinal acuity limitations. Although unattended text generally occupied somewhat more eccentric retinal positions than did attended text, the horizontal midlines of attended and unattended text were separated by only $.6^{\circ}$ of visual angle vertically. Consequently, most unattended targets occupied near-foveal vision during the reading of attended text. Research with lexical stimuli that were separated by considerably larger vertical distance has shown semantic interference and facilitation in a Stroop-type color-naming task (Kahneman \& Chajczyk, 1983). Kahneman and Chajczyk presented two words above and below a to-benamed central bar of color. Vertically, each word and the bar subtended $1^{\circ}$ and $1.4^{\circ}$ of visual angle, respectively. Even though the horizontal midlines of the two words were separated by more than $2.4^{\circ}$ of visual angle vertically, their labeling affected the naming of the central color bar. Retinal acuity limitations, therefore, cannot account for the lack of semantic target activation from unattended targets in the present study, in which vertical line separation amounted to less than $1^{\circ}$.

\section{Fixation Durations}

Our analysis of multiple-choice performance rests on two critical assumptions that warrant more detailed examination. First, we assumed a direct relationship between shifts of attention and overt eye movements to the attended region. Even though there is strong evidence in support of a functional relationship between covert and overt shifts of attention (e.g., Rayner, McConkie, \& Ehrlich, 1978), it has been shown that, under some laboratory conditions, shifts of visuospatial attention can be executed without corresponding changes in eye position (Posner, Nissen, \& Ogden, 1978). Covert shifts of visuospatial attention 
could have been directed towards the unattended line, resulting in the acquisition of useful semantic information. Occasional fixations of to-be-neglected text, therefore, could have been caused by the readers' continuous processing of it for meaning. ${ }^{2}$ According to this view, overt attention was focused at attended text and covert attention monitored to-be-neglected text. This splitattention strategy may have been used because the subjects realized that some questions on the multiple-choice test pertained to unattended text.

The relatively large difference in correct-choice performance for questions pertaining to attended and unattended text suggests, however, that subjects did not split attention into spatially distinct overt and covert components to obtain semantic information from attended and unattended text. To examine the occurrence of covert shifts of attention to unattended text more directly, we performed an analysis of cumulated viewing time on attended words that were directly above unattended targets. For instance, we analyzed the cumulated viewing time on the attended word boomerang (see Appendix), which is above the unattended target African. This cumulated viewing time was analyzed as a function of whether or not the forced-choice question corresponding to the unattended target was answered correctly. If covert shifts of attention required some measurable amount of time (Posner, 1980) and resulted in the acquisition of useful semantic information, then fixation durations on attended text should have been longer when the questions pertaining to the spatially proximal unattended target were answered correctly. The data argue against this view. Viewing time of attended text was $559 \mathrm{msec}$ when the multiple-choice questions pertaining to the spatially proximal unattended target were answered correctly and $568 \mathrm{msec}$ when the multiple-choice questions were answered incorrectly $(t<1)$.

Second, we assumed that forced-choice performance can be used to index semantic processing during the reading of attended and unattended text. However, unattended text could gain access to some type of temporary semantic representations that may no longer be accessible when multiple-choice questions are asked after passage reading has been completed. Although this possibility cannot be ruled out with certainty, two closely related aspects of the present data argue against this view: (1) Forcedchoice performance was sensitive to the semantic representation of unattended text, when unattended lines were occasionally fixated, and (2) there was no evidence in support of covert shifts of attention in the absence of overt shifts in eye position (see above paragraph).

Did overt shifts of attention to unattended text differ from overt shifts of attention to attended text? A supplementary analysis of fixation durations was designed to address this question. If readers decided to shift overt attention to unattended and attended text to obtain semantic information, fixations of unattended and attended text should be equivalent. If extra processing time was needed to encode contextually unrelated information, as occurs when a fixation of unattended text follows several prior fixations of attended text, then to-be-neglected text could require longer fixation durations than could attended text. On the other hand, shifts of attention to unattended text may have been identified as erroneous and been counteracted by an immediate redirection of attention to attended text. If this was the case, then fixation durations on unattended text should be shorter than fixation durations on attended text.

The data support the view that fixation of unattended text resulted, at least in part, from errors in attention shifting and that attention was immediately redirected to attended text. Fixation durations on unattended text averaged $179 \mathrm{msec}$ (fixation $n$ ), which was considerably shorter than the two preceding and two following fixations on attended text, which averaged $275 \mathrm{msec}$ (fixation $n-2), 314 \mathrm{msec}$ (fixation $n-1), 260 \mathrm{msec}$ (fixation $n+1$ ), and 260 msec (fixation $n+2)[F(4,220)=8.312$, $\left.M S_{\mathrm{e}}=13,494, p<.001\right]$. Paired comparisons applied to two consecutive fixations showed that fixations of unattended text were significantly shorter than were the preceding and following fixations of attended text [fixations $n-1$ and $n+1$, respectively; $t(55)=4.945, M S_{\mathrm{e}}=$ $27.2, p<.001$, and $t(55)=3.070, M S_{\mathrm{e}}=26.3$, $p<.005]$. None of the remaining comparisons were significant (all $t \mathrm{~s}<1$ ).

Two aspects of these data are noteworthy: First, shifts of attention to unattended text were immediately corrected by shifts of attention to attended text. Second, shifts of attention to the to-be-neglected line did not appear to disrupt the reading of attended text; the fixations preceding and following the fixation of to-be-neglected text remained unperturbed by the intervening "erroneous" fixation.

\section{DISCUSSION}

Our selective reading study revealed three major results: First, readers obtained useful semantic information from unattended text, as indexed by their performance in a forced-choice task that queried information from unattended passages. Second, readers inadvertently shifted attention to unattended text, as indexed by short-duration eye fixations on unattended text. Third, no useful semantic information was obtained from unattended text when it was not the recipient of inadvertent fixations.

It is unlikely that the lack of semantic activation without the direct fixation of unattended text was due to visual acuity limitations, since unattended text was "identifiable," occupying only a slightly more eccentric retinal area than attended text. Fixation of the word boomerang in the upper line of the sample passage, for instance, does not preclude the identification of the target $A$ frican shown beneath it. As stated above, the retinal disparity between attended and unattended text amounted to less than $1^{\circ}$ of visual angle, and unattended text should have occupied foveal or near-parafoveal retinal locations during the reading of attended text. 
Subanalyses of fixation durations also argue against the possibility that readers shifted attention from attended to unattended text without corresponding eye fixations. Counter to this view, fixations on attended text directly above to-be-neglected targets were nearly identical, irrespective of whether multiple-choice questions pertaining to the unattended target were answered correctly or incorrectly; fixation durations on attended text should have been longer if covert attention was shifted to unattended text below to acquire effective semantic information. Insensitivity of the forced-choice task to one-line processes could bias the results toward acceptance of the null hypothesis, but such an account of the present results also appears unwarranted, given that forced-choice performance reflected the acquisition of semantic information when attended and unattended lines of text were fixated. It is also unlikely that small differences in text-presentation modes between the present study and Willows and MacKinnon's experiments affected the use of unattended text. Again, the present results replicated Willows and MacKinnon's central finding in showing that useful semantic information was obtained from attended and tobe-neglected text in a selective-reading task.

Our central finding, that the readers did not obtain effective semantic information from to-be-neglected text in the absence of overt eye movements to neglected text, raises the question of how the readers succeeded in focusing attention at attended text and excluded the semantic processing of spatially adjacent to-be-neglected text. Several studies of visual attention indicate that subjects tend to process irrelevant stimuli, even when the processing of these stimuli is not needed for task completion. Letter-classification studies show that the classification time of attended target letters can be affected by the identity of simultaneously presented irrelevant "flanker" letters (Eriksen \& Eriksen, 1974; Grice, Boroughs, \& Canham, 1984). Analogously, Stroop-type tasks show interference effects from irrelevant stimuli that are shown adjacent to to-be-named target stimuli (Kahneman, Treisman, \& Burkell, 1983). However, flanker effects in letter-classification tasks disappear, or are greatly reduced, when visual cues are provided that identify target location prior to target presentation (Yantis \& Johnston, 1990). Analogously, flanker effects in Stroop-type word-naming tasks are greatly reduced or eliminated when the spatial location of to-be-named targets is cued prior to target presentation (Kahneman et al., 1983).

During reading, global spatial cues such as line assignment can be used to discriminate to-be-attended from tobe-neglected text. Given that relatively detailed spatial information (e.g., word length) can be gleaned up to 20 character spaces into the periphery (McConkie \& Rayner, 1975), readers may use spatial information to focus attention on a selected line of text prior to the parafoveal and foveal processing of the line's constituent words. This spatial preprocessing may serve a dual function: to in- sure the acquisition of semantic information from words occupying attended lines and to exclude the acquisition of semantic information from irrelevant words occupying flanking lines.

\section{REFERENCES}

Corteen, R. S., \& DUNN, D. (1974). Shock associated words in a nonattended message: A test for momentary awareness. Joumal of Experimental Psychology, 102, 1143-1144.

Corteen, R. S., \& WoD, B. (1972). Autonomic responses to shockassociated words in an unattended channel. Joumal of Experimental Psychology, 94, 308-313.

CoWAN, N. (1988). Evolving conceptions of memory storage, selective attention, and their mutual constraints within the human information-processing system. Psychological Bulletin, 104, 163-191.

Eriksen, B. A., Eriksen, C. W. (1974). Effects of noise letters upon the identification of a target letter in a nonsearch task. Perception \& Psychophysics, 16, 143-149.

Grice, G. R., Boroughs, J. M., CANham, L. (1984). Temporal dynamics of associative interference and facilitation produced by visual context. Perception \& Psychophysics, 36, 499-507.

HOLENDER, D. (1986). Semantic activation without conscious identification in dichotic listening, parafoveal vision, and visual masking: A survey and appraisal. Behavioral \& Brain Sciences, 9, 1-23.

Johnston, W. A., WILson, J. (1980). Perceptual processing of nontargets in an attention task. Memory \& Cognition, 8, 372-377.

KAHNEMAN, D., ChaJCZYK, D. (1983). Tests of the automaticity of reading: Dilution of Stroop effects by color-irrelevant stimuli. Journal of Experimental Psychology: Human Perception \& Performance. 9, 497-509.

Kahneman, D., Treisman, A., Burkell, J. (1983). The cost of visual filtering. Journal of Experimental Psychology: Human Perception \& Performance, 9, 510-522.

LACKNER, J. R., \& GARRETT, M. F. (1972). Resolving ambiguity: Effects of biasing context in the unattended ear. Cognition, 1, 359-372.

LEWIS, J. L. (1970). Semantic processing of unattended messages using dichotic listening. Journal of Experimental Psychology, 85, 220-227.

MAcKAY, D. G. (1973). Aspects of a theory of comprehension, memory and attention. Quarterly Journal of Experimental Psychology, 25. 22-40.

McConkie, G. W., \& RAyner, K. (1975). The span of the effective stimulus during a fixation in reading. Perception \& Psychophysics, 17, 578-586.

MORRusON, R. E. (1984). Manipulation of stimulus onset delay in reading: Evidence for parallel programming of saccades. Journal of Experimental Psychology: Human Perception \& Performance, 5, 667-682.

NewSTEAD, S. E., DENNIS, I. (1979). Lexical and grammatical processing of unshadowed messages: A reexamination of the Mackay effect. Quarerly Joumal of Experimental Psychology, 31, 477-488.

PosNer, M. I. (1980). Orienting of attention. Quarterly Journal of Experimental Psychology, 32, 3-25.

Posner, M. I., Nissen, M. J., OGden, W. C. (1978). Attended and unattended processing modes: The role of set for spatial location. In H. L. Pick \& I. J. Saltzman (Eds.), Modes of perceiving and processing information. Hillsdale, NJ: Erlbaum.

RAYNer, K., McConkIe, G. W., Ehruch, S. F. (1978). Eye movements and integrating information across fixations. Journal of Experimental Psychology: Human Perception \& Performance, 4, 529-544.

Treisman, A. (1960). Contextual cues in selective listening. Quarterly Journal of Experimental Psychology, 12, 242-248.

Treisman, A., Squire, R., Green, J. (1974). Semantic processing in dichotic listening? A replication. Memory \& Cognition, 2, 641-646.

Von Wright, J. M., Anderson, K., \& Stenman, U. (1975). Generalization of conditioned GSRs in dichotic listening. In P. M. A. Rabbitt \& S. Domic (Eds.), Attention and performance (Vol. 5, pp. 194-204). New York: Academic Press. 
Wardlaw, K. A., \& Kroll, N. E. (1976). Autonomic responses to shock-associated words in a nonattended message: A failure to replicate. Journal of Experimental Psychology: Human Perception \& Performance, 2, 357-360.

Willows, D. M., \& Mackinnon, G. E. (1973). Selective reading: Attention to the "unattended" lines. Canadian Journal of Psychology, 27, 292-304.

Y ANTIS, S., \& Johnston, J. C. (1990). On the locus of visual selection: Evidence from focused attention tasks. Journal of Experimental Psychology: Human Perception \& Performance, 16, 135-149.

ZELNICKE R, T. (1971). Perceptual attenuation of an irrelevant auditory verbal input as measured by an involuntary verbal response in a selective-attention task. Joumal of Experimental Psychology, 87, $52-56$.

\section{NOTES}

1. A supplemental analysis examined the acquisition of semantic information from unattended lines as a function of fixation location at the unattended line. We looked at instances in which the unattended target was not directly fixated and partitioned these instances into near target fixations, comprising the word immediately preceding the unattended target, and far target fixations, comprising all other fixations at unattended lines. The percent correct forced-choice performance amounted to $41.2 \%$ for near fixations and $35 \%$ for far fixations. This subanalysis suggests that readers gain more useful information pertaining to unattended targets as the distance between fixation location and target location decreases.

2. This possibility was suggested by Tram Neill.

\section{APPENDIX}

This Appendix shows an attended (uppercase) and unattended (lowercase) passage, as used in Experiment 1. This story was presented in three separate two-line presentations.

A BOOMERANG IS AN ANCIENT WEAPON USED BY THE ABORIGINES FOR the african desert dwelling fox, the fennec, is characterized

HUNTING AND WARFARE. THE NAME ORIGINATES FROM THE NATIVE by its small size and large ears. It's buff-colored with

WORD USED BY THE TURUWAL TRIBE IN NEW SOUTH WALES.

a short black-tipped tail and is mainly nocturnal.

The boomerang is used for

$$
\text { Corresponding Questions }
$$
a) games
b) warfare c) show
d) sport

It originated from what tribe
a) Tandori
b) Tongstin
c) Turuwal
d) Turnak

This tribe comes from where
a) New South Wales
b) New Zealand
c) New West Wales

Where does the fennec come from
a) Africa
b) the Americas
c) Europe
d) Asia

It has what kind of ears

a) sharply pointed b) black-tipped c) large

It has what kind of tail
a) long
b) black-tipped
c) bushy

(Manuscript received February 2, 1990;

revision accepted for publication September 10, 1990.) 